\title{
L'UTILIZZO DEL MEZZO FILMICO IN AMBITO DIDATTICO
}

\author{
Emanuela Nardo, Insegnante \\ Liceo Scientifico "M. Grigoletti" di Pordenone; \\ Ricercatrice, già docente a contratto di Pedagogia Sperimentale \\ presso l’Università agli Studi e S.S.I.S.S. di Trieste, \\ docente di Psicologia dell'educazione I.S.S.R. di Portogruaro (VE), (Italia) \\ e-mail: emanuelan@teletu.it
}

\begin{abstract}
Riass unto
Le sfide che i media pongono sul piano didattico-educativo sono molteplici; il loro influsso sulle persone in generale, e sui giovani in particolare, è potente ed innegabile, poiché i media costituiscono uno dei canali preferenziali attraverso cui conoscere la realtà circostante e formarsi su di questa un'opinione. Dal momento che gli studenti in età compresa tra 14 e 18 anni sono dei fruitori del mezzo filmico, il presente lavoro si propone di affinare strumenti che possano permettere ai docenti di utilizzare, attraverso il mezzo filmico stesso, idee pregresse dei ragazzi sugli argomenti oggetto di studio o approfondimento (Trasversalità della media education, Masterman, A. 1997). Il film, infatti, ha il potere di evocare, attraverso il ricordo, emozioni, sentimenti e ciò che più è rimasto impresso nella memoria. Attraverso un confronto tra due differenti metodi di indagine (questionario-intervista e mezzo filmico) su di una tematica coinvolgente del mondo giovanile ( l'idea di coppia in adolescenza) viene messo in evidenza non solo che tra i due strumenti possa realizzarsi una proficua sinergia, ma anche come l'uso del mezzo filmico riesce a far emergere aspetti emozionali che difficilmente con la sola intervista verrebbero fuori. La strategia metodologicoeducativa messa in atto si presta a possibili utilizzi in ambiti diversi, come viene illustrato in conclusione.
\end{abstract}

Parole chiave: Media, mezzo filmico, questionario-intervista, ricerca, confronto, metodologia didattica, adolescenza, idea di coppia.

Nessun'altra arte come il cinema va direttamente ai nostri sentimenti.

Le ombre, mute o parlanti,

si rivolgono direttamente alle segrete regioni del mio animo.

Ingmar Bergman 
Che i media abbiano influenzato a più livelli la nostra esistenza è fuori di dubbio, e se il massmediologo canadese Marshall McLuhan (1986) sosteneva che: "Le tecnologie non sono solo invenzioni che la gente utilizza ma significati che contribuiscono a reinventare le persone", è sicuramente urgente che anche la scuola fornisca degli elementi per comprendere tali strumenti.

Sono molte le sfide che i media pongono sul piano educativo. Il rapporto tra educazione e media è sempre stato complesso, per un fondamentale motivo: i fini dell'educazione (la crescita armonica e lo sviluppo dell'individuo secondo modelli e valori trasmessi) e i fini dei media (un tempo più informativi e culturali, oggi più che altro di intrattenimento e commerciali) non sono quasi mai compatibili, né è sempre possibile armonizzarli.

I media seguono le loro logiche interne, che sono prevalentemente commerciali: le conseguenze educative, psicologiche, evolutive dei messaggi non sono un problema degli autori, dei produttori televisivi, tanto meno dei pubblicitari.

Ciò che conta, invece, per chi si occupa di educazione, sono soprattutto gli effetti che $\mathrm{i}$ media producono, al di là dell'intenzionalità o della previsione di tali effetti.

Per quanto concerne gli aspetti educativi e formativi che mi interessa analizzare, occorre partire dalla considerazione che i media esercitano una innegabile e potente influenza sulle persone ${ }^{1}$, in particolare su quelle in età evolutiva. Ciò avviene per il semplice fatto di contribuire a determinare l'ambiente in cui le persone (compresi i bambini) vivono e apprendono. Non solo: i media occupano gran parte del tempo libero delle persone, costituiscono la primaria fonte di informazione sul mondo e la società civile, contribuiscono a formare e consolidare idee e convinzioni, condizionano i consumi e gli stili di vita, determinano il consenso e orientano la pubblica opinione, quindi hanno effetti diretti sulla qualità della democrazia. I media riguardano dunque moltissimi aspetti del nostro vivere quotidiano. Utilizzare la lente dell'educazione, cioè analizzare la presenza dei media nei suoi aspetti educativi e formativi è non solo utile, ma urgente, e, mi permetto di dire, attività troppo poco praticata.

Nella mia prassi educativa e nell' ambito della ricerca qui presentata, mi sono occupata di come la fruizione del mezzo filmico da parte degli studenti possa permettere ai docenti di utilizzare idee pregresse circa argomenti oggetto di studio o approfondimento (Grollo M., Nardo E., 2007). Il film ha il potere di evocare, attraverso il ricordo, emozioni e sentimenti e ciò che più è rimasto impresso nella memoria. E' uno strumento mediatico capace di porre nella condizione di poter pensare a un tema o ad un argomento partendo dalle emozioni. Ripensare a ciò che è stato visto rende possibile sviluppare una capacità di lettura critica rispetto a quanto vediamo, rendendoci consapevoli che ci colpiscono delle immagini più di altre

${ }^{1}$ Tra i volumi che si riferiscono alle diverse posizioni possiamo citare la riedizione di Popper (2000) sugli effetti negativi della televisione e viceversa il volume di Morcellini (1999), che presenta una visione più positiva e propositiva. 
perché queste sollecitano in noi con il nostro significato, delle riflessioni. In questo modo ci sembra che quello spezzone di film possa esprimere bene un certo concetto, in quanto veicola l'immagine che noi abbiamo di quel dato argomento.

Bene o male, tutti noi siamo cultori, o quantomeno fruitori, di storie raccontate mediante un film; anche solo il saper scegliere, tra le innumerevoli immagini che ci bombardano, quelle che maggiormente ci hanno colpito potrebbe rappresentare una preziosa fonte di informazioni "indirette" ma alquanto credibili.

Ecco dunque l'idea di coinvolgere un gruppo di studenti, con i quali utilizzare il mezzo filmico per veicolare argomenti di cui altrimenti sarebbe difficile parlare. Per sfruttare le conoscenze filmiche pregresse dei ragazzi, ho predisposto una serie di materiali atti a realizzare un'indagine dal vero, per individuare problemi e vissuti tipici dell'adolescenza. In questa sede presenterò estratti di una ricerca sull'idea di coppia in adolescenza. Metterò in evidenza i risultati diversi che si ottengono utilizzando due differenti strumenti didattici: il questionario-intervista e i film. Evidenzierò in coda alla ricerca come la metodologia didattica del mezzo filmico possa essere impiegata dai docenti con finalità diverse.

\section{Indagine sull'idea di coppia in adolescenza}

\section{Domanda di ricerca}

"Gli adolescenti ritengono che la coppia genitoriale da cui provengono influenzi la loro idea di coppia?"

\section{Obiettivi}

1. indagare sull'idea di coppia che gli adolescenti hanno;

2. capire quali aspetti della propria coppia genitoriale gli adolescenti utilizzano come punto di riferimento per formare un propria coppia;

\section{Soggetti}

46 studenti liceali (26 maschi e 20 femmine) di età compresa tra i 17 e i 18 anni frequentanti tre classi $4^{\wedge}$ di un Liceo Scientifico di Pordenone, anno scol. 200809 , che, ai fini della ricerca, vengono conglobati in un unico gruppo.

\section{Tipologia di analisi}

Si è scelto di effettuare una ricerca qualitativa (Celi F. e Fontana D., 2003) piuttosto che quantitativa, in quanto la finalità principale consiste nel fatto di condurre un'indagine per studiare il pensiero adolescente.

Questa ricerca si serve inoltre di un disegno quasi-sperimentale (McBurnay D.H., 1994), poiché viene utilizzato un gruppo precostituito (gruppo classe) non potendo isolare a priori le variabili che intervengono nella situazione da studiare. Infine, non si vogliono generalizzare i risultati ottenuti, in quanto non è possibile controllare tutte le differenze individuali e contestuali che potrebbero influenzare l'esito della ricerca ogni volta in modo diverso. 


\section{Fasi}

La ricerca è fondamentalmente costituita di tre fasi:

1. Fase di preparazione: dopo una ricerca teorica sull'idea di coppia in adolescenza e sui fattori che possono influire sull'identità dei figli adolescenti, sono stati strutturati appositamente degli strumenti per studiare l'idea di coppia in adolescenza, e il tipo di influenza che i ragazzi sentono dai loro genitori, per formare una propria coppia.

Si è posta particolare attenzione nell'utilizzare strumenti che non fossero troppo invasivi, data l'età dei ragazzi, ma che permettessero, in qualche modo, di stimolare la loro attenzione e la loro creatività.

2. Fase di somministrazione degli strumenti: gli strumenti predisposti sono stati somministrati agli studenti in unica seduta.

\section{Fase di elaborazione dei risultati.}

\section{Strumenti}

La predisposizione degli strumenti ha tenuto conto dell'età dei ragazzi, da un lato, sufficientemente matura per poter operare una auto-riflessione, ma non sufficientemente adulta per lasciar campo libero a delle domande troppo personali. Gli strumenti utilizzati in questa ricerca sono stati fondamentalmente due:

\section{Questionario-intervista}

E' strutturato in 5 domande di varia tipologia:

- La prima domanda richiede di scegliere 5 delle 12 parole elencate, che i ragazzi ritengono più importanti ( $1=$ più importante, $5=$ meno importante), per rilevare l'idea di coppia che essi hanno. Tali parole sono state successivamente raggruppate post hoc nelle seguenti categorie, da me create: ideale sentimentale, ideale valoriale, componente temporale, attenzione all'altro, componente egoistica, repulsione.

Le parole sono presentate in modo casuale.

- La seconda domanda richiede di nominare liberamente 3 parole, e serve per rilevare l'idea che hanno gli adolescenti circa le cause di rottura di una coppia della propria età.

- La terza domanda richiede di nominare liberamente 3 parole al fine di rilevare l'idea che hanno gli adolescenti circa le cause di rottura di una coppia di genitori.

- La quarta domanda richiede ai ragazzi di esprimere, attraverso una valutazione da 0 (meno importante) a 5 (più importante), quanto i genitori rappresentino un riferimento perché essi possano costruire una propria idea di coppia solida.

- La quinta domanda, la cui risposta va scelta tra tre opzioni (per niente, in parte, molto), chiede se il fatto di avere dei genitori separati, possa, a parere dei ragazzi, influenzare negativamente la loro idea di coppia.

2. Mezzo filmico 
Attraverso il mezzo filmico viene richiesto ai ragazzi di scegliere dei film, ed in particolare qualche scena, che riporta alla mente il loro concetto di "coppia ideale".

Successivamente, viene richiesto loro di descrivere le emozioni trasmesse da tale scena e che cosa vorrebbero comunicare nel momento in cui dovessero farla vedere a qualcuno (genitori, insegnanti, coetanei, al proprio partner).

Infine, viene data la consegna di riunirsi in gruppi di 3 o 4 persone ed attraverso una scheda appositamente predisposta, di selezionare tra i film scelti due soli spezzoni condivisi da tutto il gruppo, motivando tale scelta.

\section{Risultati del questionario relativo a "l'idea di coppia"}

\section{Quesito ${ }^{\circ} 1$ :}

Quali delle seguenti parole ti fanno pensare all'idea di coppia?

Viene chiesto ai ragazzi di scegliere 5 delle 12 parole elencate, e di numerarle da 1 a 5 in ordine di importanza ( $1=$ più importante, $5=$ meno importante). A ciascuna risposta viene attribuito un punteggio che poi si traduce in percentuale di gradimento. I risultati sono riportati nel grafico 1.

\begin{tabular}{|l|}
\hline \multicolumn{1}{|c|}{ PAROLE DA } \\
SCEGLIERE
\end{tabular}

Sembrerebbe, dai dati raccolti e dalle conseguenti percentuali, che per gli adolescenti sia nel complesso molto importante in una coppia prioritariamente l'aspetto "ideale-sentimentale", rappresentato dall'amore e dalla fedeltà, e, subito dopo, la componente "egoistica", che ricerca un'affermazione del proprio io attraverso il sentirsi considerati, accettati, amati. Parimenti, la categoria "attenzione all'altro" riscuote numerosi consensi, a testimonianza dell'importanza per i giovani di condividere ogni cosa per potersi sentire uniti. Restano invece in subordine gli aspetti "valoriali" (legame e famiglia) e quelli "temporali" (occasionale e definitivo) e "repulsivi" (obbligo e costrizione). In sintesi, l'idea che emerge dai giovani è 
quella di una coppia in cui valori come l'amore, la fedeltà, il sentirsi accolti e la condivisione, che nella società odierna sembrano in controtendenza, vengono percepiti come fondamentali.

GRAFICO 1 "Quali delle seguenti parole ti fanno pensare all'idea di coppia?"

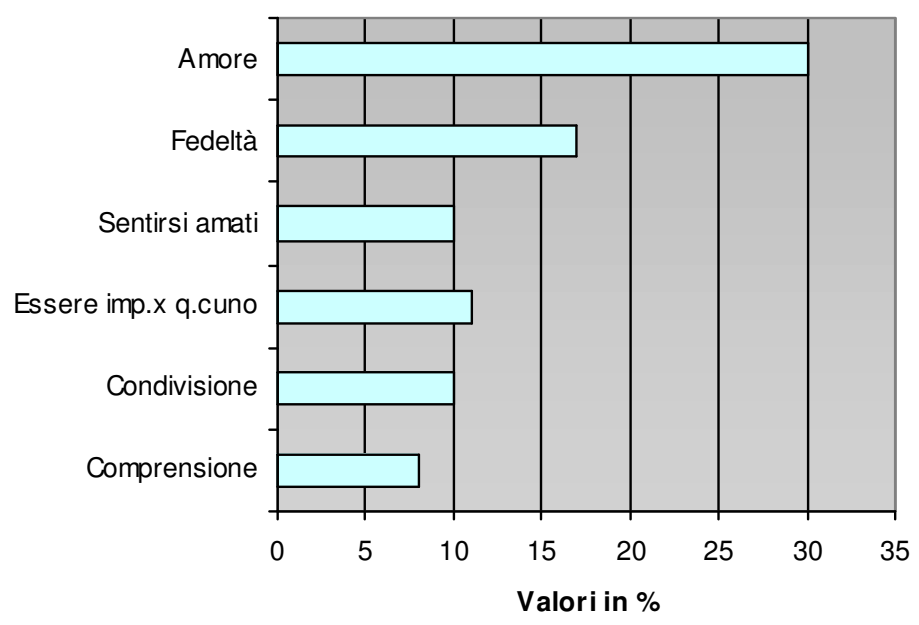

Quesiti $n^{\circ} 2$ e $n^{\circ} 3$ :

Quali sono a tuo parere $i$ tre principali fattori che possono provocare la rottura di una coppia della tua età (di una coppia di genitori)?

In queste due domande viene richiesto ai ragazzi di elencare liberamente 3 fattori che, a loro parere, possono provocare la rottura, rispettivamente, di una coppia della loro età (quesito 2), e di una coppia di genitori (quesito 3).

A ciascuna delle parole riportate dagli intervistati sono stati attribuiti dei punteggi, corrispondenti al numero degli eventi, e quindi un valore percentuale, rispetto agli eventi totali.

Per esigenze di sintesi, nel grafico 2 sottostante vengono analizzati e comparati, per le due domande, solamente i fattori che hanno ottenuto un maggior punteggio.

Il dato che emerge prepotentemente da questo confronto è il fatto che, sia nella percezione di una coppia di adolescenti che di genitori, l'infedeltà, e quindi la presenza di una terza persona, rappresenti di gran lunga il principale motivo di rottura. Al secondo posto, troviamo per entrambi i tipi di coppia l'incomprensione; evidentemente, i giovani percepiscono la fatica del capirsi e del farsi capire nella coppia, a prescindere dall'età. Mentre tuttavia nelle coppie di coetanei la fine dell'amore e i litigi non vengono ritenuti una causa di rottura frequente, queste risultano nelle coppie di genitori elementi sicuramente percepiti come più 
determinanti. Per contro, l'importanza attribuita alla voce trascuratezza nelle giovani coppie testimonia il bisogno di sentirsi protetti, amati e curati, molto più che nelle coppie di genitori, dove spesso subentra la routine.

Quesito ${ }^{\circ} 4$ :

Quanto $i$ genitori costituiscono un riferimento affinché $i$ propri figli adolescenti possano costruire una solida idea di coppia?

In questo quesito viene attribuita una valutazione da 0 (non vi è alcun riferimento) a 5 (sono un riferimento). Le votazioni da 3 a 5 vengono considerate una risposta affermativa al quesito; da 0 a 2 , una risposta negativa. I dati sono stati poi convertiti in valore percentuale. In questo caso sono state distinte le risposte date dalla componente femminile e da quella maschile degli intervistati, come risulta dal grafico 3 sottostante.

Emerge che per entrambi i sessi i genitori sono complessivamente un valido riferimento per i ragazzi. Tuttavia le valutazioni sono state date maggiormente dai maschi che dalle femmine. Ciò sembra dimostrare che le ragazze, rispetto ai ragazzi, prendono meno spunto dalla coppia genitoriale per formare una propria idea di coppia. La valutazione " $0 / 1 / 2$ " (negativa), dove le votazioni femminili superano di ben due volte quelle maschili, lo confermerebbe.

Complessivamente, quindi, in riferimento al campione considerato, sembrano essere i maschi, rispetto alle femmine, a basarsi maggiormente sulla coppia genitoriale.

GRAFICO 2 "Confronto tra coppia adolescente e coppia di genitori"

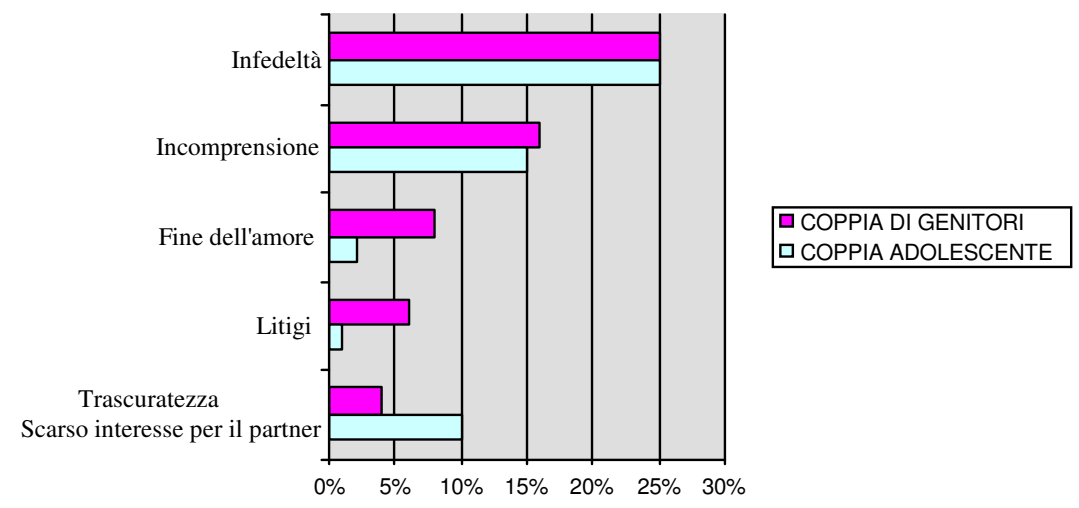

Quesito ${ }^{\circ} 5$ :

A tuo avviso, avere i genitori separati può influenzare negativamente l'idea di coppia di un ragazzo/a della tua età?

In questo quesito i ragazzi dovevano attribuire una delle seguenti valutazioni: molto, in parte, per niente. Anche in questo caso, il numero degli eventi per ciascuna 
voce è stato convertito in valore percentuale e i risultati sono stati espressi nel grafico 4.

Come si osserva, la valutazione "in parte" risulta di gran lunga la più utilizzata, seguita da "molto" e con soltanto meno di un quarto degli intervistati, da "per niente". Questo dato non può non confermare l'idea che i genitori costituiscono un valido riferimento al fine di costruire una solida idea di coppia, siano essi separati o meno.
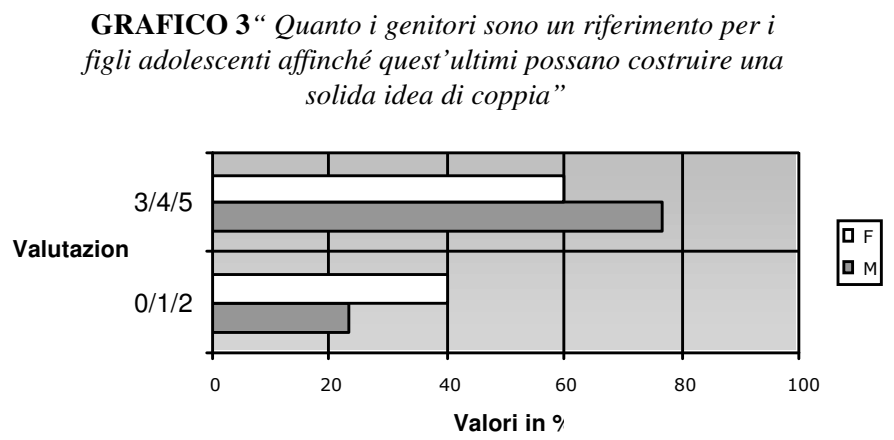

GRAFICO 4 "A tuo avviso, avere $i$ genitori separati, può influenzare negativamente l'idea di coppia di un ragazzo/a della tua età?’

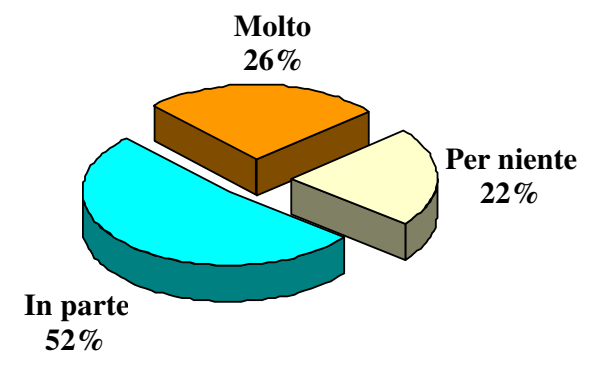

\section{Risultati del mezzo filmico relativo a "L'idea di coppia nei film"}

Viene inizialmente somministrata ai ragazzi una scheda (si veda la scheda A alla pagina seguente), dove viene richiesto di scegliere uno, o al massimo due film, ed in particolare la scena che meglio esprime la loro immagine di "coppia ideale" ("Scegli la scena di un film da te visto, che esprima al meglio la tua immagine di "coppia ideale"). 
Dall'analisi degli elementi raccolti mediante tale scheda, emergono le emozioni trasmesse dalle scene scelte, "l'idea di coppia" che ne deriva e i destinatari a cui i ragazzi farebbero vedere i film.

\begin{tabular}{|c|c|c|}
\hline & LISTA FILM PROPOSTI & $\begin{array}{l}\text { FILM SCELTI } \\
\text { IN GRUPPO }\end{array}$ \\
\hline \multirow{3}{*}{ GRUPPO 1} & Il diavolo veste Prada & \\
\hline & Romeo e Giulietta & $\mathbf{X}$ \\
\hline & Titanic & $\mathbf{X}$ \\
\hline \multirow{4}{*}{ GRUPPO 2} & Il diavolo veste Prada & \\
\hline & Shakespeare in love & \\
\hline & Titanic & $\mathbf{X}$ \\
\hline & Tre metri sopra il cielo & \\
\hline \multirow{4}{*}{ GRUPPO 3} & Ghost & \\
\hline & Drumline & \\
\hline & 40 giorni e 40 notti & $\mathbf{X}$ \\
\hline & Taking off & \\
\hline \multirow{4}{*}{ GRUPPO 4} & American Pie & \\
\hline & Tre metri sopra il cielo & $\mathbf{x}$ \\
\hline & Ho voglia di te & \\
\hline & Romeo e Giulietta & \\
\hline \multirow{4}{*}{ GRUPPO 5 . } & Ghost & $\mathbf{X}$ \\
\hline & Manuale d'amore & \\
\hline & Donny Darke & \\
\hline & Edward mani di forbice & $\mathbf{X}$ \\
\hline \multirow{3}{*}{ GRUPPO 6} & La bella e la bestia & \\
\hline & Shakespeare in love & \\
\hline & Flyboys & $\mathbf{X}$ \\
\hline \multirow{4}{*}{ GRUPPO 7 } & Scrivimi una canzone & \\
\hline & Save the last dance & $\mathbf{X}$ \\
\hline & Big Fish & \\
\hline & C'è posta per te & \\
\hline \multirow{3}{*}{ GRUPPO 8} & Pretty, Princess & \\
\hline & La sirenetta & $\mathbf{X}$ \\
\hline & Ghost & $\mathbf{X}$ \\
\hline \multirow{3}{*}{ GRUPPO 9} & Big Fish & $\mathbf{x}$ \\
\hline & American Pie & \\
\hline & 40 giorni e 40 notti & $\mathbf{X}$ \\
\hline \multirow{3}{*}{ GRUPPO 10} & Romeo e Giulietta & \\
\hline & Mr and Mrs Smith & $\mathbf{x}$ \\
\hline & Manuale d'amore 2 & \\
\hline \multirow{4}{*}{ GRUPPQ 11} & Aladin & $\mathbf{X}$ \\
\hline & Biancaneve e i 7 nani & \\
\hline & Shrek II & $\mathbf{X}$ \\
\hline & Un'ottima annata & $\mathbf{x}$ \\
\hline \multirow{2}{*}{ GRUPPO 12} & II Signore degli anelli (libro 1) & $\mathbf{X}$ \\
\hline & Il diavolo veste Prada & $\mathbf{x}$ \\
\hline \multirow{2}{*}{ GRUPPO 13} & I passi dellamore & $\mathbf{X}$ \\
\hline & Romeo and Juliet & $\mathbf{X}$ \\
\hline \multirow{3}{*}{ GRUPPO 14} & Tre metri sopra il cielo & $\mathbf{X}$ \\
\hline & La vita secondo Jim & \\
\hline & Titanic & $\mathbf{x}$ \\
\hline \multirow{2}{*}{ GRUPPO 15} & American Pie & $\mathbf{x}$ \\
\hline & Titanic & $\mathbf{x}$ \\
\hline
\end{tabular}

Nella fase successiva i ragazzi si sono organizzati in gruppi da 3-4 persone, per selezionare 2 film condivisi da tutto il gruppo, giustificando la motivazione di 
tale scelta. Nella tabella 1 sono elencati i film scelti dai ragazzi, per esprimere la loro immagine di coppia ideale. Nella $2^{\wedge}$ colonna sono, altresì, evidenziati i film scelti dopo la discussione di gruppo, dove emergono sia i film votati, sia i film scartati. Non verranno commentate tali votazioni, ma solo le motivazioni che hanno indotto a tali scelte.

Con gli spezzoni selezionati, si è poi proceduto al montaggio di un filmato da mostrare agli intervistati. L'utilizzo del mezzo filmico, doveva, nell'intento dello Tabella 1 sperimentatore, favorire gli aspetti più emotivi relativi all'idea di coppia che gli adolescenti hanno, rispetto ad uno strumento diverso e più razionaleanalitico, come il questionario-intervista.

\section{SCHEDA A: "L'idea di coppia nei films"}

Scegli la scena di un film da te visto, che esprima al meglio la tua immagine di "coppia ideale".

Titolo dei films scelti ( al massimo due):

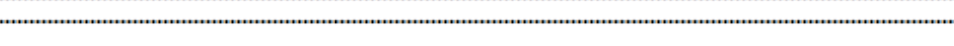

Spezzone da:

Descrivi la scena spiegandone il contenuto:

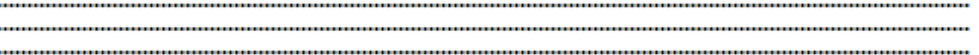

Secondo te, che "idea di coppia" emerge in questa scena?

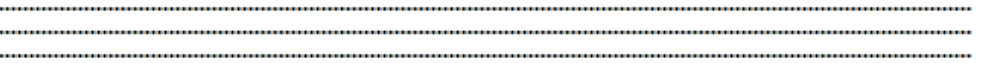

Che emozioni ti trasmette?

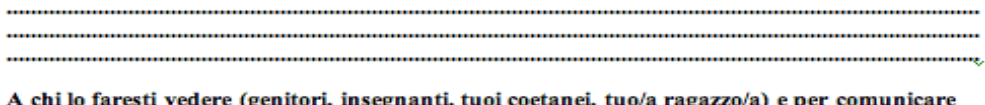
che cosa?

Analizziamo le risposte fornite dal campione in relazione alle emozioni trasmesse dalla scena selezionata.

Per l'analisi dei risultati sono state utilizzate le parole usate dai ragazzi; sono state poi conglobate le emozioni che risultavano esser simili.

Nella tabella 2, per maggior chiarezza, sono state riportate le prime 5 parole più votate.

Verranno pertanto commentati solamente $\mathrm{i}$ dati che hanno ottenuto un maggiore "punteggio".

Come si può osservare, la maggior parte dei ragazzi preferisce film che trattano tematiche d'amore, raggiungendo complessivamente 12 voti totali. Lo 
stesso vale per i film dove emerge la Felicità/Pace/Serenità (con 8 voti totali). Non manca di certo la componente malinconica (5 voti), tipica dell'adolescenza e della Tenerezza/Dolcezza (5 voti) che coinvolge anche la componente maschile.

\begin{tabular}{|l|c|c|c|}
\hline & M & F & TOT \\
\hline Amore & 7 & 5 & $\mathbf{1 2}$ \\
\hline Felicità/Pace/Serenità & 3 & 5 & $\mathbf{8}$ \\
\hline Passione & 6 & & $\mathbf{6}$ \\
\hline Malinconia & 3 & 2 & $\mathbf{5}$ \\
\hline Tenerezza/Dolcezza & 2 & 3 & $\mathbf{5}$ \\
\hline Fiducia/Fedeltà & 1 & 2 & 3 \\
\hline Voglia di vivere & 1 & 2 & 3 \\
\hline Complicità & & 2 & 2 \\
\hline Sicurezza/Conforto & 1 & 1 & 2 \\
\hline Entusiasmo & & 1 & 1 \\
\hline Curiosità & & 1 & 1 \\
\hline Libertà & & 1 & 1 \\
\hline Sensibilità & & 1 & 1 \\
\hline
\end{tabular}

Infine il dato che a mio avviso colpisce maggiormente, è la Passione la quale è stata "gettonata" solo ed esclusivamente dai maschi (6 voti). Questa componente emerge con una certa decisione, aspetto che non era stato tenuto presente nell'elaborazione del questionario. Infine sono emerse anche altre emozioni non meno importanti, come la "Voglia di vivere", la "Sensibilità", la "Libertà", la "Curiosità", il bisogno di "Sicurezza/Conforto", la "Fiducia", l'"Entusiasmo" e il Tabella 2 desiderio di "Complicità".

Analizziamo ora le risposte fornite dal campione in relazione all'idea di coppia veicolata dalla scena. Nel grafico 5 sono elencate le idee di coppia più ricorrentemente nominate dai ragazzi. Per convenienza, sono state utilizzate delle categorie post hoc.

Le percentuali sono state calcolate sul numero complessivo del gruppo (40 persone, 20 maschi e 20 femmine, dato che 6 maschi non hanno risposto). Le percentuali sono state arrotondate al numero intero.

Dall'analisi di questo grafico, che riporta l'idea di coppia che hanno i ragazzi attraverso i film, emerge che circa 1/3 del gruppo ha idealizzato una Coppia disposta a tutto per amore (e che lotta ogni giorno per stare insieme). In secondo luogo è stata votata una Coppia stabile, serena e in continua crescita $(25 \%)$. Sembra che la continua lotta per stare insieme, il raggiungimento di un equilibrio e la maturazione, siano degli aspetti fondamentali nella coppia ideale degli adolescenti.

Tuttavia, un aspetto che a mio avviso riveste una importanza notevole, e che emerge più chiaramente nel grafico 7 , risiede nel fatto che il $13 \%$ abbia scelto una 
Coppia unita dall'attrazione, a testimonianza del fatto che, l'affettività e la sessualità sono due dimensioni strettamente connesse soprattutto in adolescenza, in quanto un compito di sviluppo fondamentale per tutta la vita è proprio la capacità di instaurare con persone del sesso opposto relazioni affettive e sessuali stabili. Altro modello importante considerato è la Coppia unita dalla complicità, che ha totalizzato il $13 \%$ delle votazioni.

Per concludere, analizziamo le risposte alla domanda: A chi lo faresti vedere (genitori, insegnanti, tuoi coetanei, tuo/a ragazzo/a)?

I destinatari a cui i ragazzi farebbero vedere i film scelti sono raggruppati nella Tabella 3. E' stata aggiunta la voce "Tutti" durante l'analisi dei dati, poiché gran parte dei ragazzi l'ha preferita alle alternative date.

Le percentuali sono state calcolate sul numero complessivo del gruppo (42 persone, dato che 4 non hanno risposto), distinguendo tuttavia la componente maschile da quella femminile per effettuare un confronto. Anche in questo caso per semplicità le percentuali sono state arrotondate al numero intero.

Un dato interessante consiste nel fatto che nonostante l'argomento sia alquanto delicato, il 33\% del gruppo farebbe vedere il film scelto al proprio ragazzo/a, e che quindi circa 1/3 del gruppo riesce a parlare liberamente della propria idea di coppia. C'è da sottolineare che tale Tabella 3: "A chi faresti vedere il film scelto?" scelta è stata fatta prevalentemente dalla componente femminile $(40 \%)$ rispetto a quella maschile (27\%), e questo sembra delineare una maggiore maturità sessuale da parte delle ragazze.

Anche il 36\% del gruppo sarebbe disposto a far vedere i film scelti $a$ qualsiasi persona, e ciò non esclude che anch'essi riescano a padroneggiare tale argomento.

\begin{tabular}{|l|c|c|c|}
\hline & M & F & TOT \\
\hline Tutti & $45 \%$ & $25 \%$ & $36 \%$ \\
\hline Ragazzo/a & $27 \%$ & $40 \%$ & $33 \%$ \\
\hline Coetanei & $23 \%$ & $35 \%$ & $29 \%$ \\
\hline Genitori & $5 \%$ & $0 \%$ & $\mathbf{2} \%$ \\
\hline Insegnanti & $0 \%$ & $0 \%$ & $0 \%$ \\
\hline
\end{tabular}

Altro dato importante, è il $29 \%$ dei ragazzi che ha scelto come destinatario del messaggio $i$ coetanei, come se fosse ancora vincolato agli amici. Il gruppo di pari è l'inizio di una possibile società alternativa, non copiata dagli adulti, ma originariamente diversa. E' il gruppo che consente all'individuo di provare e sperimentare. La ribellione dai genitori non è solo un desiderio di autonomia, ma un vero bisogno di innovazione. Solo che questa innovazione non viene compiuta dall'individuo o dalla coppia, ma dalla collettività giovanile. 
Viene però il momento in cui l'individuo è sufficientemente forte, sufficientemente autonomo per distanziarsi dal gruppo, dal suo giudizio, dalla sua pressione, per riprendere la strada della costruzione di una coppia amorosa.

Ultimo, ma non meno importante, il $2 \%$ del gruppo che ha scelto come destinatari $i$ genitori, o come se non avesse ancora rivendicato la propria autonomia sulla famiglia di origine, o per far capire qualcosa ai genitori. Tuttavia tale scelta è stata effettuata prevalentemente dai maschi $(5 \%)$ come se, a differenza delle femmine, avessero ancora bisogno di una base sicura.

\section{Considerazioni finali sull "idea di coppia" emersa dal confronto degli strumenti utilizzati}

Dai risultati ottenuti attraverso lo strumento del mezzo filmico, emerge una conferma di quanto già risultava dall'analisi dei risultati del questionario. In altre parole sembra si delinei per gli adolescenti, ancora una volta, un'idea di coppia basata fondamentalmente sull'amore.

Tuttavia, durante la fase di costruzione del questionario non erano state considerate delle componenti assai importanti, che invece successivamente sono emerse attraverso il mezzo filmico: la complicità e la componente sessuale (passione/attrazione). I ragazzi ritengono infatti che si tratti di due aspetti fondamentali nella coppia ideale. L'affettività e la sessualità permettono non solo di superare il proprio Sè narcisistico, ma aiutano l'adolescente ad avere una maggiore stima di sè ed una maggiore fiducia nelle proprie capacità.

Ciò nonostante, l'utilizzo del mezzo filmico non evidenzia la componente egoistica (sentirsi amati/essere importanti per qualcuno) che, invece, occupa una posizione considerevole nei risultati del questionario. Sembra che gli adolescenti fatichino molto ad ammettere il proprio bisogno di sentirsi amati e considerati da qualcuno.

\section{Considerazioni di ordine didattico}

A posteriori, dunque, se dovessi ricreare gli strumenti da somministrare, includerei sicuramente la componente sessuale, dedicando particolare attenzione all'aspetto della passione/attrazione; cercherei, inoltre, di enfatizzare l'aspetto relativo alla complicità, dato che una percentuale rilevante di soggetti ha indicato questa come una componente assai importante nell'idea di coppia degli adolescenti.

In conclusione, dunque, l'allestimento di idonei strumenti didattici per il rilevamento delle idee degli studenti circa argomenti per loro di particolare coinvolgimento non è cosa semplice e spesso occorre rielaborare, modificare, intrecciare tecniche differenti in modo che gli strumenti utilizzati possano implementarsi in un meccanismo a feedback. In questo processo, per scardinare le difese personali e le reticenze a manifestrare le proprie intime emozioni il mezzo 
filmico può davvero rappresentare un valido supporto didattico. L'utilizzo del mezzo filmico, in effetti, può essere applicato in vari contesti:

- per introdurre, approfondire un argomento;

- per analizzare una tematica da più prospettive;

- per favorire una discussione;

- per comprendere in modo non invasivo il vissuto degli adolescenti;

- per avere indicazioni per predisporre strumenti di indagine più mirati;

- per favorire la metariflessione.

Il mezzo filmico quindi, può essere utilizzato in vari modi, sia in ambito didattico che in ambito educativo; personalmente ho potuto seguire ricerche condotte con giovani di centri di aggregazione giovanile, ad esempio per indagare sulla loro idea di divertimento e per predisporre attività e servizi a loro adeguati.

Gli ambiti in cui ricerche mediante l'utilizzo dei films sono state da me condotte e curate con adolescenti rivestono tematiche diverse come: paure ed emozioni dell'adolescenza nei film, l'idea di divertimento attraverso films, il livello di accettadione della diversità (culturale, sessuale, mentale, fisica), il rapporto con il corpo. In questa sede si è voluto presentare fondamentalmente uno strumentario ad uso degli insegnanti. I risultati delle ricerche ed il confronto tra i due differenti strumenti di indagine mi hanno rassicurata circa l'effettiva validità didatticoeducativa dell'utilizzo del mezzo filmico, che riesce a far emergere aspetti che difficilmente con la sola intervista sarebbero venuti a galla.

\section{Bibliografia} Trento.

CELI F., FONTANA D., (2003), "Fare ricerca sperimentale a scuola", ed. Erickson,

CIGOLI V., GIULIANI C., IAFRATE R., (2002), Il dolore del divorzio: adolescenti e giovani tra riavvicinamento e distacco alla storia famigliare, in Psicologia Clinica dello Sviluppo. Pag. 423-442, a. VI, n.3, dicembre. Carocci.

COGGI C., RICCHIARDI P., (2005), Progettare la ricerca empirica in educazione, Roma,

GROLLO M. NARDO E., (2007), "Educare con i Media dalle competenze orizzontali alla consapevolezza. Proposte e progetti di educazione ai media" ed. Junior Bergamo.

MC BUMAY D.N., (1994), Metodologia della ricerca in psicologia, Bologna, Il Mulino.

MC LUHAN M.,(1986), Gli strumenti del comunicare, Garzanti, Milano.

NARDO E., (2007), "Avete sempre ragione voi! manuale di sopravvivenza per adulti e adolescenti”, ed. Erickson, Trento.

RIVOLTELLA P.C., (2001), Media education. Modelli, esperienze, profilo disciplinare. Carocci, Roma. 
ZAVATTINI G.C., et altri, (2002), Rappresentazione della famiglia negli stili di attaccamento in adolescenti di famiglie unite e separate, in Psicologia Clinica dello Sviluppo. Pag. 491-514, a. VI, n.3, dicembre. 